\title{
Link Scheduling for Exploiting Spatial Reuse in Multi-hop MIMO Networks
}

\author{
Deke Guo, Member, IEEE, Yuan He, Member, IEEE, Yunhao Liu, Senior Member, IEEE, \\ Panlong Yang, Member, IEEE, Xiangyang Li, Senior Member, IEEE, Xin Wang, Member, IEEE
}

\begin{abstract}
MIMO has great potential for enhancing the throughput of multi-hop wireless networks via spatial multiplexing or spatial reuse. Spatial reuse with stream control (SC) provides a considerable improvement of the network throughput over spatial multiplexing. The gain of spatial reuse, however, is still not fully exploited. There exist large numbers of additional data streams, which could be transmitted concurrently with those data streams scheduled by stream control at certain time slots and vicinities. In this paper, we address the issue of MIMO link scheduling to maximize the gain of spatial reuse and thus network throughput. We propose a receiveroriented interference suppression model (ROIS), based on which we design both centralized and distributed link scheduling algorithms to fully exploit the gain of spatial reuse in multi-hop MIMO networks. Further, we address the traffic-aware link scheduling problem by injecting non-uniform traffic load into the network. Through theoretical analysis and comprehensive performance evaluation, we achieve the following results: (1) Link scheduling based on ROIS achieves significant higher network throughput than that based on stream control, with any interference range, number of antennas, and average hop length of data flows. (2) The traffic-aware scheduling is enticingly complementary to the link scheduling based on ROIS model. Accordingly, the two scheduling schemes can be combined to further enhance the network throughput.
\end{abstract}

Index Terms-Spatial reuse, stream control, MIMO, scheduling, wireless multi-hop networks

\section{INTRODUCTION}

MIMO (Multiple-Input-Multiple-Output) has often been referred to as a solution to increase the network throughput of wireless networks. Many efforts have been made to deal with single-user MIMO communications [1]. Multiple antennas yield the degree-of-freedom (DOF) gain. The additional DOFs can be fully exploited by spatial multiplexing multiple independent data streams onto one MIMO channel, hence resulting in increased network capacity without extra bandwidth or power cost. Recently, the co-channel interference in multi-user MIMO communications [2] draw considerable attention from the community, such as the broadcast channel (BC) [3] and the multiple-access channel (MAC) [4].

In multi-hop MIMO networks [5], some efforts have been made in recent years on developing MAC and routing protocols, e.g., [6], [7], [8], [9]. In such a setting, MIMO enhances the potential network throughput via spatial multiplexing by achieving high data rates or spatial reuse of the spectrum by

- D. Guo is with the Key laboratory for Information System Engineering, College of Information System and Management, National University of Defense Technology, Changsha 410073, P.R. China. E-mail: guodeke@gmail.com.

- Y. He is with the TNLIST and the School of Software, Tsinghua University, Beijing 100084, P.R. China. E-mail: heyuan@cse.ust.hk.

- Y. Liu is with the School of Software, TNLIST, Tsinghua University, and the Hong Kong University of Science and Technology.E-mail: liu@cse.ust.hk.

- P. Yang is with the Institute of Communication Engineering, P.L.A. University of Science and Technology, Nanjing 210007, P.R. China. E-mail: panlongyang@gmail.com.

- X. Li is with Department of Computer Science, IIT, and is a visiting professor of School of Software Engineering, Dalian University of Technology. E-mail: xli@cs.iit.edu.

- X. Wang is with the College of Electronic Science and Engineering, National University of Defense Technology, Changsha 410073, P.R. China. E-mail:wxwireless@nudt.edu.cn. allowing multiple simultaneous communications at the same vicinity. In the case of spatial reuse, each MIMO link only employs partial DOFs to transmit and receive data streams. Basically there are three approaches. The first one is the spatial reuse with stream control (SC) [6], [10], [11], [12], [13], as discussed in Section 2.3. The best DOFs are selected for data transmissions, while the other DOFs at the receiver are used to suppress interfering data streams. In the second approach, referred to as the spatial reuse with non-stream control, DOFs for data transmissions are randomly selected while the receiver uses the other DOFs in the same way as the first approach [14], [15]. The third approach is referred to as the spatial reuse with interference avoidance [14]. The transmitter selects the appropriate DOFs to transmit data streams such that the other DOFs can null its data streams at undesired nearby receivers, which are receiving intended streams from other transmitters.

Existing work have revealed that stream control increases the total link throughput by $20-65 \%$ for a set of mutually interfering links [10], [11]. In [8], [16], the authors demonstrate that stream control improves the overall throughput compared to the TDMA-based spatial multiplexing. In [12], [13], the authors point out that stream control with the optimal antenna selection is an attractive alternative to spatial multiplexing. Moreover, stream control is effective in a wide range of wireless environments, while spatial multiplexing exhibits its benefits only under rich scattering or strong multi-path conditions associated with urban and indoor applications. The network throughput when the stream control is used, however, is still considerably constrained.

Bearing these points in mind, we address the issue of link scheduling to exploit the gain of spatial reuse in multihop MIMO networks, and to enhance the potential network throughput. The challenges of such a work include how to deal 
with the interference effect so that we can increase the number of transmission streams at the same time slot, frequency slot, and vicinity. Our studies show that while stream control outperforms the TDMA-based spatial multiplexing, the gain of spatial reuse is still not fully approached and can be further exploited by using interference avoidance. To address such a problem, we model the spatial reuse mechanism as a link scheduling problem, which incorporates both stream control and interference avoidance. We also present efficient centralized and distributed link scheduling algorithms. The main contributions of this paper are summarized as follows.

1) We propose the receiver oriented interference suppression (ROIS) model to deal with interference. Each MIMO link with data to be transmitted is scheduled by stream control first, or by interference avoidance if stream control cannot assign the smallest time slot to it.

2) We design both centralized and distributed link scheduling algorithms to optimize the network throughput. Further, we design traffic-aware link scheduling algorithms to meet the traffic demands. The improved algorithms can significantly enhance the network throughput.

3) Through comprehensive simulations, we show that the link scheduling based on ROIS provides a significant improvement of the network throughput than that based on stream control. The traffic-aware link scheduling achieves the largest network throughput.

The rest of this paper is organized as follows. In Section 2 we describe the background of our work. Section 3 introduces the network model and the link scheduling problem. We elaborate the centralized link scheduling algorithms in Section 4. We propose the distributed and traffic-aware link scheduling algorithms in Sections 5 and 6, respectively. The evaluation results are reported in Section 7. We review the related work in Section 8, and conclude this paper in Section 9.

\section{Background and Preliminaries}

\subsection{Single-user MIMO communication}

In rich scattering environments, a non-selective block-fading MIMO channel with $m$ transmitting and $n$ receiving omnidirectional antennas can be modeled by a $n \times m$ channel matrix $H$. The $n$-dimensional signal at the output of the receiving antennas is $y=H x+a$, where $x$ accounts for the $m$-dimensional transmitted symbols, and $a$ denotes the $n \times 1$ additive white Gaussian noise vector. Let $h_{i j}$ represent the gain of the channel from a transmitting antenna $j$ to a receiving antenna $i$.

At the transmitter side, an encoder with the channel coding, interleaving, and symbol-mapping blocks is responsible for producing the input symbol vector, $x_{1}, \ldots, x_{m}$. The transmitter directly delivers the symbol vector to the receiver from respective antennas. At the receiver side, number of $n$ signals are received, each signal $y_{i}$ being a linear combination of the $m$ transmitted symbols plus an additive noise where $1 \leq i \leq n$. That is $y_{i}=\sum_{j=0}^{m} h_{i j} \times x_{j}+a_{i}$.

\subsection{Transceiver model}

In this paper, we focus on the MIMO transceiver model $z=W(H M x+a)$ that includes the linear pre-processing and

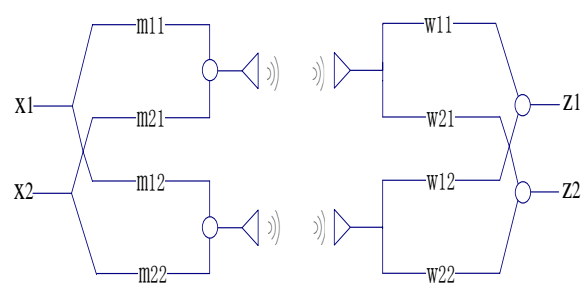

Fig. 1. An example of MIMO processing.

the post-processing performed at the transmitter and receiver, respectively. The actual transmitted signals $H M x$ are generated using an $m \times m$ pre-processing matrix $M$ in which each column is referred to as a transmitting weight vector for a symbol of $x$. As shown in Fig.1, number of $m=2$ weighted copies of each symbol in $x$ are produced and sent to the $m$ transmit antennas, one on each antenna. A mixed signal of $m$ weighted symbols is produced at each transmitting antenna. The $m$ mixed signals are then delivered to an intended receiver through a MIMO channel $H$. The received vector of signals is $H M x+a$, and is converted into an estimation of the original vector signal $x$ by a $n \times n$ post-processing matrix $W$ in which each row is referred as a receiving weight vector.

If $H$ is perfectly known at the transmitter, a MIMO channel with $m$ transmitting antennas and $n$ receiving antennas can be decomposed into $\min \{m, n\}$ independent parallel subchannels. In such a case, channel capacity is achieved by using linear precoding known to be capacity-optimal where $M$ and $W$ are the right and left singular vectors of $H$, respectively.

\subsection{Spatial reuse with interference}

If stream control and spatial multiplexing transmit the same number of data streams at a time slot, stream control outperforms the latter in terms of the network throughput. The root cause is that although a MIMO channel can be decomposed into multiple parallel sub-channels, their capacities have quite large disparities for moderate or low SNR [8], [11], [17].

As shown in Fig.2, each node has four antennas. Consider transmissions from node $A$ to node $B$ along link 1 , and from node $C$ to node $D$ along link 2 . Since links 1 and 2 are close to each other, they encounter mutual interference. If the TDMAbased spatial multiplexing is adopted, only one transmission is allowed to take place at any slot while the transmission may proceed with all the four streams. There is a channel gain for each stream, denoted as the stream gain. As aforementioned, these stream gains are usually not equal. In the above example, the normalized gains of $1,0.9,0.7$, and 0.6 are assumed on the four streams of link 1 , while that of $1,0.85,0.8$, and 0.6 are assumed on the four streams of link 2 .

As for the spatial reuse with stream control, the two best sub-channels with gains 1 and 0.9 are selected for link 1, while the two best sub-channels with gains 1 and 0.85 are selected for link 2. Consequently, stream control provides an improvement of around $20 \%$ than the TDMA-based spatial multiplexing. Nevertheless, such a result is not true for the spatial reuse with non-stream control. Without careful selection of the DOFs, the non-stream control approach might choose the worse sub-channels for data transmissions. 

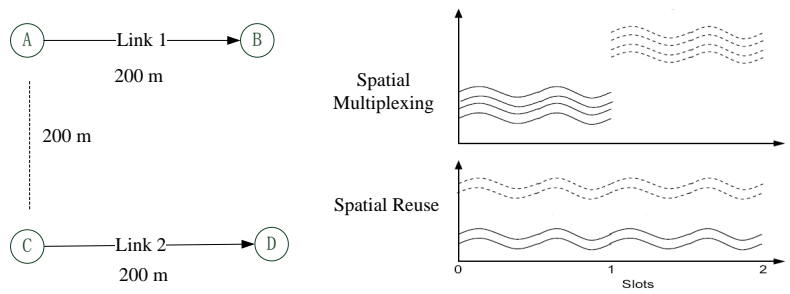

Fig. 2. Examples of spatial multiplexing and spatial reuse.

As for the spatial reuse with interference avoidance, node $A$ uses stream control or non-stream control to deliver two data streams over link 1 since no streams are currently receiving by nearby nodes within $A$ 's interference range. Node $C$ nulls its signals at interfered receiver $B$ prior to transmitting its two data streams, namely directing signal energy towards the intended node $D$ and minimizing interference to node $B$. Although such an approach also transmits four data streams per time slot, the resultant network throughput, however, is less than that of stream control. The cause is that it is not necessary that the transmission over link 2 employs the best two sub-channels.

\section{SYSTEM MODEL AND ASSUMPTIONS}

We start with a brief introduction to the network model and propose an interference suppression model for multi-hop MIMO networks and then model link scheduling problem.

\subsection{Network model}

We consider a multi-hop wireless network with homogeneous terminals deployed in a rich scattering environment. Each terminal has $m$ antennas, following the aforementioned transceiver model. The communication graph is modeled as a directed graph $G=(V, L)$ with a vertex set $V$ and an edge set $L$. A vertex of the graph corresponds to a terminal with $m$ antennas. A directed edge $(u, v)$ denotes a MIMO link $l$, through which $v$ can correctly decode the received signal from $u$. Here $u$ and $v$ are referred to as the transmitter $t(l)$ and receiver $r(l)$ of $l$, respectively.

Each vertex $u$ has an associated transmission range (decode range), denoted by $d_{t}(u)$. A necessary but not sufficient condition for a transceiver $v$ to hear $u$ is that $v$ is within the Euclidean distance $d_{t}(u)$ from $u$. Edge $(u, v) \in L$ if and only if $v$ is within the distance $d_{t}(u)$ from $u$. We assume that all vertices have the identical transmission power, thus identical transmission range. Each vertex $u$ has an interference range $d_{i}(u)$ such that vertex $v$ is interfered by $u$ only if $v$ resides within a distance $d_{i}(u)$ of $u$. Typically, $d_{i}(u)>d_{t}(u)$. The interference ranges of all nodes are also assumed to be identical. Given a set of pairs of source and destination, the end-to-end flow for each pair of nodes can be discovered by existing routing protocols [18], thus determining the corresponding communication graph.

\subsection{Interference suppression model}

Two different types of interference are the primary interference and the secondary interference [19]. The primary interference also refers to the radio constraint, a radio either transmits or

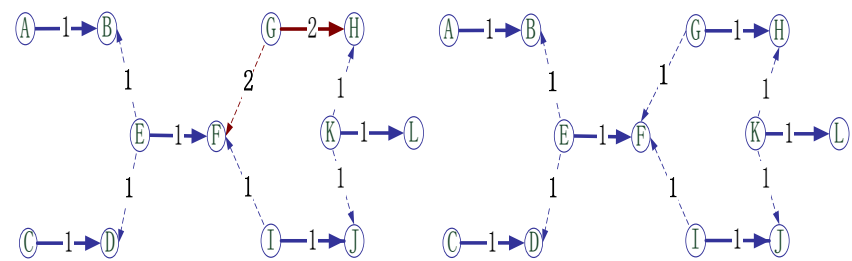

(a) Stream control model

(b) ROIS model

\section{Fig. 3. Examples of interference suppression models.}

receives, but cannot transmit and receive at the same time. The secondary interference occurs when multiple separate streams reach the same receiver, thus the receiver observes a composite signal of these streams and noise. We focus on two interference suppression models, including the stream control (SC) model and a new model proposed in this paper.

In the case of stream control, each transmitter employs the linear precoding technology to pursue the highest data rates for data streams, while does not intend to reduce interference to other receivers interfered by it. That is, each receiver with $m$ antennas can suppress interference caused by undesired nearby transmitters while successfully receiving desired signals, as long as it does not observe more than $m$ data streams. The receiver that observes more than $m$ streams cannot decode its desired streams. As shown in Fig.3(a), terminals $B, D$, and $J$ can decode their desired streams from terminals $A, C$, and $I$ at time slot 1 , respectively. Terminal $F$, however, cannot decode its desired streams from terminal $E$ if link $l_{G H}$ is also active at time slot 1 such that it has two antennas while observes three streams. Link $l_{G H}$ is scheduled to be active at time slot 2 .

After performing stream control at a given time slot $t$, consider any unscheduled link $l$ which does not satisfy the constraint of stream control at that time slot. That is, the receiver of at least one active link at time slot $t$ has observed $m$ streams if that link is interfered with link $l$. Link $l$, however, has a chance to be active at time slot $t$ if it adopts the interference avoidance technique. In this way, the transmitter of link $l$ can null its signals at those nearby receivers observing $m$ non-nulling streams, while makes its receiver decode the intended signal. Here, a non-nulling stream observed by a terminal means that the transmitter of that stream does not null its signals at such a terminal. We name such a procedure as the ROIS model, which appropriately combines stream control and interference avoidance approaches for maximizing the number of concurrent stream transmissions.

In summary, the basic idea of the ROIS model is that each MIMO link with data to be transmitted is scheduled by stream control first, or interference avoidance if stream control cannot assign the smallest time slot to it. Thus, for the receiver of any active link, all observed streams are non-nulling if it observes no more than $m$ streams. Otherwise, additional streams besides the $m$ non-nulling streams are nulling streams. Here, no matter a receiver observes how many nulling streams, it can extract all $m$ non-nulling streams after canceling such nulling streams.

\subsection{Problem formulation}

A schedule of a set of links describes the specific time slots, at which packets are moved over the links. Let $X_{l, t}$ be an 
indicator variable, $X_{l, t}=0$ only if link $l$ is inactive and no packet is sent over it in time slot $t$, and $X_{l, t}=i>0$ if link $l$ is active and $i$ concurrent streams are sent over it in time slot $t$. We employ a periodic schedule with a period length of $T$ such that $X_{l, j T+t}=X_{l,(j+1) T+t}$ for any time slot $t$ and integer $j$. For any two links $u$ and $v$, link $u$ interferes with link $v$ only if the transmission on link $u$ interferes with the reception on link $v$. However, this does not necessarily imply that link $v$ also interferes with link $u$. Let $I_{l}^{+}$denote the set of links whose receivers are interfered by the transmission on link $l$ at the same time slot, and let $I_{l}^{-}$denote the set of links whose transmissions interfere with the reception on link $l$.

Given a communication graph $G=(V, L)$, we have to derive a feasible schedule for $L$ such that the network throughput is maximized, i.e., with the smallest $T$. Thus, at least one stream can be delivered over each link $l \in L$ in every $T$ time slots. A feasible schedule for $L$ has to satisfy the constraints imposed by the primary and secondary interference.

According to the primary interference, a necessary condition to schedule link $l$ at time slot $t$ is that

$$
X_{l^{\prime}, t}^{t}=0, \forall l^{\prime} \in\left\{l^{\prime} \mid l^{\prime} \in L, t(l)=r\left(l^{\prime}\right) \text { or } r(l)=t\left(l^{\prime}\right)\right\} .
$$

That is, each terminal of link $l$ either transmits or receives, but cannot transmit and receive at the same time.

Consider that any link $l \in L$ can be scheduled to be active, according to stream control or interference avoidance. To avoid confusion, we use $X_{l, t}^{s c}$ and $X_{l, t}^{r s}$ to denote the number of concurrent streams sent over link $l$ at time slot $t$ under stream control and interference avoidance, respectively. Note that $X_{l, t}^{s c}$ and $X_{l, t}^{r s}$ should not exceed zero simultaneously since each transceiver works under either steam control or interference avoidance at a given time slot. Let $N_{i}(l, t)$ and $N_{u}(l, t)$ denote the number of intended and unintended streams observed by the receiver of link $l$, respectively. Let $N(l, t)$ denote the number of total streams observed by the receiver of link $l$ at time slot $t$, where $N(l, t)=N_{i}(l, t)+N_{u}(l, t)$.

For the secondary interference, we focus on two interference suppression models, i.e., the SC and ROIS models. Given a communication graph $G$, we will consider the precondition for a feasible schedule under the two models, respectively.

According to the secondary interference under the SC model, we have

$$
\begin{aligned}
& N_{i}(l, t)=\sum_{l^{\prime} \in L, r\left(l^{\prime}\right)=r(l)} X_{l^{\prime}, t}^{s c} \\
& N_{u}(l, t)=\sum_{l^{\prime} \in I_{l}^{-}, r\left(l^{\prime}\right) \neq r(l)} X_{l^{\prime}, t}^{s c} .
\end{aligned}
$$

In such a case, a receiver with $m$ antennas can decode all received non-nulling streams together (at most $m$ ), or just those intended streams after suppressing all unintended streams as interference. Thus, we can derive Theorem 1 as follows.

Theorem 1: A schedule $S$ for $L$ is feasible under the SC model only if it is feasible at any time slot $t \in T$, i.e., formulas (1) and (2) hold for each active link $l \in L$ at time slot $t$.

$$
N(l, t) \leq m
$$

According to the secondary interference under the ROIS model, there is

$$
\begin{aligned}
& N_{i}(l, t)=\sum_{l^{\prime} \in L, r\left(l^{\prime}\right)=r(l)} X_{l^{\prime}, t}^{s c}+X_{l^{\prime}, t}^{r s} \\
& N_{u}(l, t)=\sum_{l^{\prime} \in I_{l}^{-}, r\left(l^{\prime}\right) \neq r(l)} X_{l^{\prime}, t}^{s c}+X_{l^{\prime}, t}^{r s} .
\end{aligned}
$$

According to the basic idea of ROIS model, we can derive Theorem 2 as follows.

Theorem 2: A schedule $S$ for $L$ is feasible under the ROIS model only if for any time slot $t \in T$, active links scheduled by the SC model is feasible under the SC model, and Formula (3) holds prior to each active link $l$ scheduled by the interference avoidance model.

$$
\left\{\begin{array}{l}
\sum_{l^{\prime} \in I_{l}^{+}, N\left(l^{\prime}, t\right) \geq m} N_{i}\left(l^{\prime}, t\right)<m \\
N(l, t)<m
\end{array}\right.
$$

Formula (3) involves the following two conditions.

1) Among all receivers interfered by link $l$, the total number of intended streams at those receivers, each of which has observed $m$ non-nulling streams and possible nulling streams, is less than $m$. That is, at least one of $m$ DOFs is available for transmitting streams over link $l$, while others are used to null its streams at the receptions of the intended streams for its interfered receivers.

2) The number of streams observed by the receiver of link $l$ should be less than $m$ prior to the transmission of link l. Otherwise, the receiver of link $l$ cannot decode its upcoming intended streams since all observed streams are scheduled when link $l$ is inactive, hence being nonnulling streams.

As shown in Fig.3(b), links $l_{A B}, l_{C D}, l_{E F}, l_{I J}$, and $l_{K L}$ are active at time slot 1 . In the $S C$ model, a link $l_{G H}$ should not be active at time slot 1 since a node $F$ (one neighbor of the node $G$ ) has observed $m=2$ streams with two antennas. In the ROIS model, the link $l_{G H}$ can be active at time slot 1 if the node $G$ nulls its signals at the node $F$ using the interference avoidance technique. The two conditions of Formula 3 hold for the link $l_{G H}$. First, the link $l_{G H}$ interferes one receiver $F$ which observes two streams, including one intended stream. Second, the receiver of link $l_{G H}$ only observes one stream from the node $K$. Thus, the node $G$ can use one DOF for transmitting a stream over the link $l_{G H}$ and another one for nulling its transmission at the node $F$.

\section{Centralized Link Scheduling}

We first define an interference graph to characterize the special constraints of MIMO link scheduling. We then present the centralized link scheduling algorithms for the SC and ROIS models, respectively.

\subsection{Interference graph}

Given a communication graph $G$, the conflict graph has been widely used to represent the interference in $G$. There is an edge between two vertices in a conflict graph if the two corresponding links in the communication graph conflict due to interference and cannot be active simultaneously [20]. It, however, cannot characterize the special constraints of MIMO link scheduling as discussed in Section 3. First, it does not 
distinguish the radio constraint and the wireless interference such that two link suffering the radio constraint appear to be two interfering links. Second, it uses undirected edges to represent interference in $G$, hence, does not characterize the difference between $I_{l}^{+}$and $I_{l}^{-}$for any link $l \in L$.

The above two issues are essential to schedule MIMO links. Accordingly, we define an interference graph, $F_{G}$, to model the effect of radio constraint and wireless interference in a communication graph $G$ of a multi-hop MIMO network. Each vertex $x y$ of $F_{G}$ corresponds to a directed link $(x, y)$ in $G$. For any two vertices $x y$ and $p q$, if $y=p$ or $q=x$, there is an undirected edge between $x y$ and $p q$ in $F_{G}$. Otherwise, there is a directed edge from $x y$ to $p q$ in $F_{G}$ if the transmission on link $(x, y)$ interferes with the reception on link $(p, q)$ in $G$, or a directed edge from $p g$ to $x y$ in $F_{G}$ if the transmission on link $(p, q)$ interferes with the reception on link $(x, y)$ in $G$. An undirected edge in $F_{G}$ indicates that a pair of links in $G$ suffer the radio constraint, thus cannot be active simultaneously. A directed edge in $F_{G}$ indicates two interfering links, which may be active simultaneously if the constraints defined in Theorem 1 or 2 can be satisfied.

For simplicity, we use a single character to label a vertex in $F_{G}$, and define basic concepts for $F_{G}$ used in the rest of this paper. The set of vertices adjacent to vertex $p q$ through an undirected edge is called the conflict-neighbors of vertex $p q$, denoted as $c_{n}(p g)$. The set of vertices incident on $p q$ is called the in-neighbors of vertex $p q$. Similarly, the set of vertices incident from $p q$ is called the out-neighbors vertex $p q$. The in-degree and out-degree of $p q$, denoted as $d^{+}(p g)$ and $d^{-}(p g)$, are the cardinality of its in-neighbors and outneighbors, respectively.

\subsection{Overview of centralized link scheduling}

The link scheduling problem has been reduced to the vertex coloring of a conflict graph, when each terminal uses only one antenna and one channel. In this paper, we focus on a different link scheduling problem facing the distinct features of MIMO links and associated interference suppression models. If each terminal has one antenna, the link scheduling problem in this paper can be treated as the vertex coloring, which is one of the thoroughly studied NP-hard problems in graph theory [19]. Therefore, we can derive that the link scheduling problem in this paper is NP-hard. We thus propose two dedicated link scheduling approaches to approximate the optimal solution.

The basic idea of link scheduling is to sort all links and then to assign each link the smallest available time slot such that a feasible link scheduling is achieved. The sorting and assigning orders of links are two important factors that affect the scheduling result. Our centralized scheduling algorithms will sort links in two special orders. The first one is as follows: we pick a vertex with the smallest in-degree in the remaining interference graph $F_{G}$, and then remove the picked vertex and its associated edges from the graph; repeat such a process until the graph is empty, as shown in the SortLinks function in Algorithm 1. The second order is similar, but each time it picks the vertex with the smallest out-degree in the remaining graph. Our scheduling algorithms then assign links in the reverse

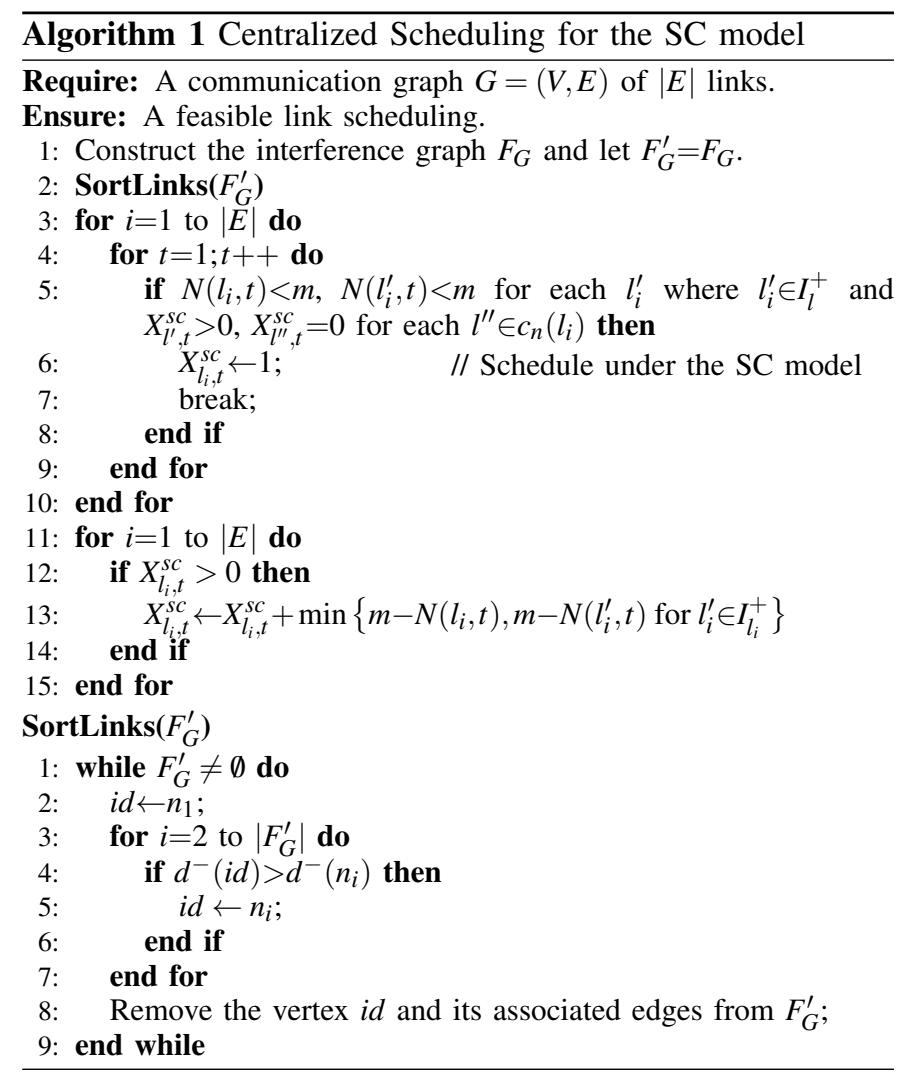

order of the sorting order. Consequently, a link with larger in-degree or out-degree will likely to be processed earlier by our algorithms. The centralized scheduling algorithms under the SC and ROIS models use the first order to sort links. Yet, they can also use the second order to sort links.

\subsection{Link scheduling under the SC model}

For the link scheduling under the SC model, Algorithm 1 first achieves the assigning order, denoted as $l_{1}, l_{2}, \ldots, l_{|E|}$, of links by invoking the SortLinks algorithm. It then processes links from $l_{1}$ to $l_{|E|}$, and assigns each $l_{i}$ the time slot $t=1$ if the following conditions hold prior to the transmission of $l_{i}$. First, none of conflict-neighbors of $l_{i}$ is active at time slot $t$. Second, the receiver of each active out-neighbors of $l_{i}$ at time slot $t$ observes less than $m$ streams at that time slot, and so does the receiver of $l_{i}$. Note that those unscheduled links will be processed in the same way after incrementing the time slot by one until all links are scheduled.

Note that an active link is initially allowed to transmit only one stream, so as to make more links be active at the same time slot, hence maximizing the gain of spatial reuse. After all links in $L$ become active, each active link $l_{i} \in L$ is appended additional streams if exist, as shown in lines 8-10 in Algorithm 1 , so as to enhance the link throughput. The largest number of additional streams over an active link $l_{i}$ with $X_{l_{i}, t}^{s c}=1$ at time slot $t$ is given by

$$
\min \left\{m-N\left(l_{i}, t\right), m-N\left(l_{i}^{\prime}, t\right) \text { for } l_{i}^{\prime} \in I_{l_{i}}^{+}\right\} .
$$

\subsection{Link scheduling under the ROIS model}

The link scheduling under the ROIS model will be accomplished by two successive stages at each time slot. Algorithm 


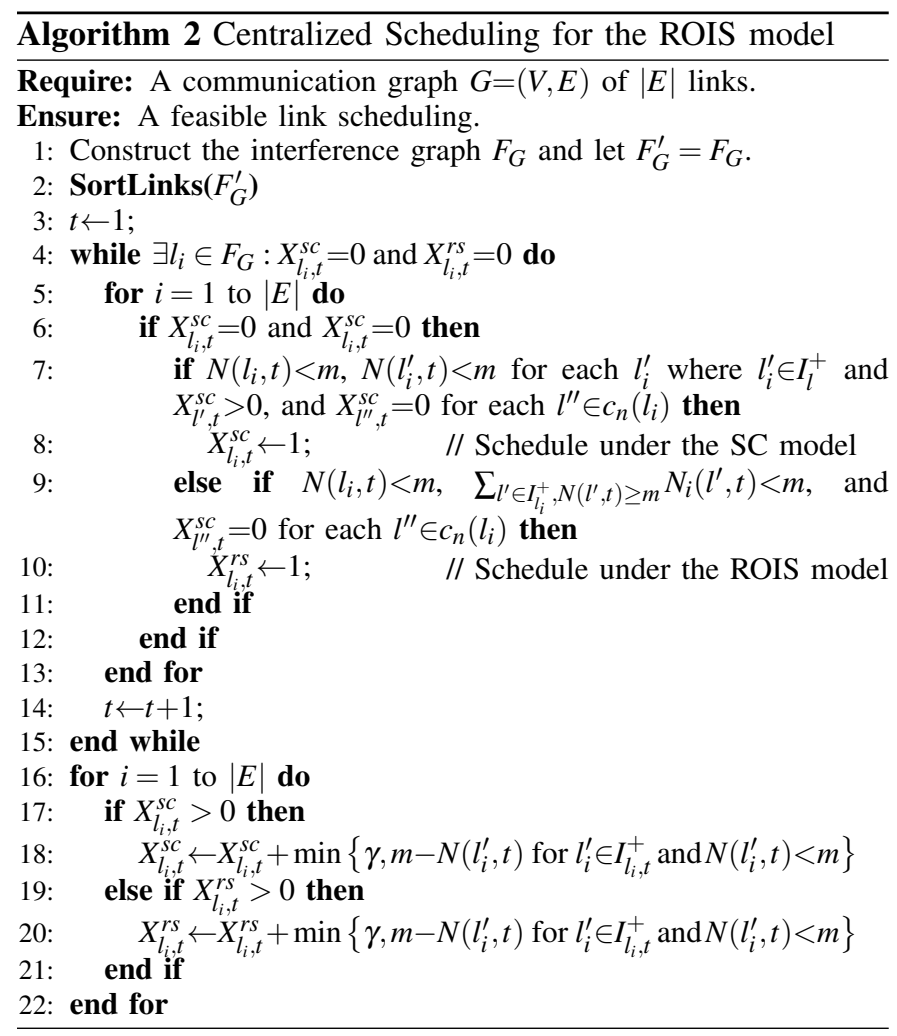

2 will process links from $l_{1}$ to $l_{|E|}$. If one link $l_{i}$ has not been scheduled, it first tries to allocate the time slot $t$ to it such that a feasible schedule under the SC model is achieved at that time slot. If the precondition of the SC model is not satisfied for that link, it will assign the link $l_{i}$ the same time slot $t$ under the interference avoidance model if the following conditions hold prior to the transmission of $l_{i}$. First, none of conflict-neighbors of $l_{i}$ are active at the time slot $t$. Second, Formula (3) holds for $l_{i}$ and $t$. If unscheduled links still exist after such a round, the process repeats in the next time slot.

Similar to Algorithm 1, an active link at a given time slot is initially allowed to transmit only one stream in Algorithm 2. It also tries to append additional streams over each active when all links have been scheduled to be active, so as to enhance the throughput of each active link, as shown in lines 12-16 in Algorithm 2. For any active link $l_{i}$ at time slot $t$, if it is scheduled by the stream control model, the largest number of additional streams over it is given by Formula (4). Otherwise, according to inequation (3) the largest number of additional streams over link $l_{i}$ should not exceed

$$
\gamma=\min \left\{m-N\left(l_{i}, t\right), m-\sum_{l_{i}^{\prime} \in I_{l_{i}}^{+}, N\left(l_{i}^{\prime}, t\right) \geq m} N_{i}\left(l_{i}^{\prime}, t\right)\right\} .
$$

This ensures that the transmitter of $l_{i}$ can null its streams at undesired nearby receivers observing $m$ non-nulling streams and possible nulling streams, while the receiver of $l_{i}$ can decode its intended streams. We find that Formula (5) is not enough to ensure a feasible scheduling. For example, terminal $C$ is initially scheduled to be active by the interference avoidance model and deliver only one stream over link $l_{C E}$ at time slot $t$, as shown in Fig.4.(a). We can see that the stream over link $l_{C E}$ is observed as a nulling stream by terminals $A$ and $B$ but an unintended stream by terminal $D$. If terminal $C$ appends $\min \{5-3,5-2\}=2$ streams over link $l_{C E}$ according to

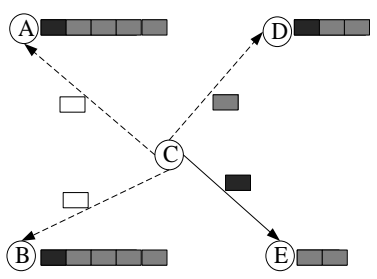

(a) one initial stream over link $\mathrm{C}->\mathrm{E}$ - Intended stream

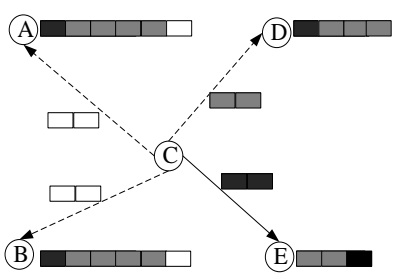

(b) two additional streams over link C->E $\square$ Nulling stream $\square$ Unintended stream

Fig. 4. Examples of link scheduling under the ROIS model, where each terminal has five antennas.

Formula (5) at that time slot, terminal $D$ no longer recovers its intended stream since it will observes six non-nulling streams, as shown in Fig.4.(b). Accordingly, given an active link $l_{i}$ at time slot $t$, each active link, which is interfered by link $l_{i}$ and observes less than $m$ streams, should observe no more than $m$ streams after appending additional streams over link $l_{i}$. Consequently, the largest number of additional streams over link $l_{i}$ with $X_{l_{i}, t}^{r s}=1$ at time slot $t$ is

$$
\min \left\{\gamma, m-N\left(l_{i}^{\prime}, t\right) \text { for } l_{i}^{\prime} \in I_{l_{i}, t}^{+} \text {and } N\left(l_{i}^{\prime}, t\right)<m\right\} .
$$

For example, the largest number of additional streams over link $l_{C E}$ should be one not two, as shown in Fig.4.

\section{Distributed LiNK SCHEdULING}

In multi-hop wireless networks, centralized algorithms are often difficult to implement; hence, we further design distributed algorithms to provide a feasible schedule. Intuitively, we can design the distributed versions for Algorithms 1 and 2. However, finding the link with the global maximum in-degree or out-degree in $F_{G}$ iteratively is not trivial. In addition, the node having packets to transmit over a link does not know the in-neighbors or out-neighbors of that link in advance, no matter the knowledge of $F_{G}$. Thus, our distributed scheduling does not process links in a specific order.

For the distributed link scheduling under the SC model, each node schedules its links according to the SC model if the links have packets to transmit, and collects information about link scheduling results from interfering nodes. In the case of the ROIS model, each node schedules its links according to the SC model or the interference avoidance model if the SC model cannot assign the smallest time slot to its links. A common process of the SC and ROIS models is to provide each node with necessary information about interfering nodes. This process involves two types of control messages. The first type is the color message, which records the time slot assigned to a link represented by a pair of transmitter and receiver. The second type is the overload message omes, which indicates that a node receives $m$ messages of type color and records the number of intended streams received.

Each node $v_{i}$ with unscheduled links or an overload message accesses the channel using one of the random access control mechanisms, such as the carrier sense mechanism and the IEEE 802.11 MAC protocol. Due to the fact that there are so many MAC mechanisms in the literature, this paper does not focus on the selection and details of those MAC protocols. If node $v_{i}$ successfully accesses the channel, it assigns 
one unscheduled link (if exists) with the smallest time slot; generates a color message and broadcasts it with an overload message (if exists) to all interfering nodes. If no unscheduled link exists, node $v_{i}$ only broadcasts the overload message. If node $v_{i}$ fails or need not to compete channel, it monitors the channel for receiving omes and/or color messages from all interfering nodes. An overload message is generated if node $v_{i}$ has received $m$ messages of type color at the time slot $t$.

Unlike the centralized algorithm, the distributed algorithm neither sorts links nor processes links in a specific order. All nodes compete the chance of scheduling its links randomly and make the scheduling decisions only based on the received control messages. The broadcast of control messages makes all related nodes achieve the necessary information to allocate the smallest time slot for each link. For any node $v_{i}$ occupying the wireless channel, one of its unscheduled link is scheduled at the current smallest time slot by stream control first, or by interference avoidance if stream control cannot assign the current smallest time slot to it. If the necessary and sufficient conditions of stream control as well as interference avoidance are not held at the current smallest time slot for node $v_{i}$, it will try to schedule such a link in the next time slot. In this way, our distributed algorithm ensures a feasible schedule in general scenarios, and is easy to be implemented without much additional computation overhead on each node.

Inspired by our centralized algorithms, each scheduled active link may have the opportunity to transmit more streams. Such opportunity can be captured if another process is appended at the end of our distributed algorithm to allocate additional streams as many as possible for each scheduled link. All necessary information for a transmitter to determine how many additional streams over a link can be obtained in the same way as Algorithms 1 and 2.

\section{LINK SCHEDULING WITH TRAFFIC LOAD}

We previously assume that the number of packets that need to go through each communication link is identical. Such an assumption, however, is not always true in reality.

Let us consider an example of multi-hop MIMO networks, as shown in Fig.5(a), in which each node has $m=2$ antennas. Assume there are $k$ flows, with flow $f_{i}$ starting at a source node $s_{i}$ and ending at a destination node $d_{i}$ for $1 \leq i \leq k$. In Fig.5(a), we can see that the transmission of link $v_{2} d_{i}$ will interfere the reception of any link with $v_{1}$ as the receiver. Thus, at least $k+1$ time slots are required to implement a feasible link schedule, which can be obtained by assigning time slot $\lceil i / 2\rceil$ to link $s_{i} v_{1}$ for $1 \leq i \leq k$, time slot $k / 2+1$ to link $v_{1} v_{2}$, and time slot $\lceil i / 2\rceil+k / 2+1$ to link $v_{2} d_{i}$ for $1 \leq i \leq k$, as shown in Fig.5(c). In such a schedule, only one stream is transmitted over links $s_{i} v_{1}$ and $v_{2} d_{i}$, whereas at most two streams can be transmitted over link $v_{1} v_{2}$. We assume that the rate at which data can be sent over links $s_{i} v_{1}$ and $v_{2} s_{i}$ is $a$ bps and that of link $v_{1} v_{2}$ is $2 a$ if each link uses all time slots. It is obvious that all $k$ flows go through link $v_{1} v_{2}$, and node $v_{1}$ will receive $k a$ data and can send only $2 a$ data every $k+1$ slots. Thus, link $v_{1} v_{2}$ becomes the bottleneck and the overall network throughput is $\frac{2 a}{k+1}$ bps. Consequently, for each flow, its throughput is approximately

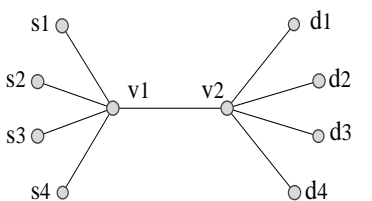

(a) network

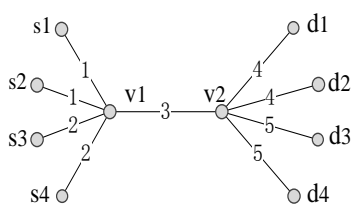

(c) unweighted link schedule, $\mathrm{m}=2$

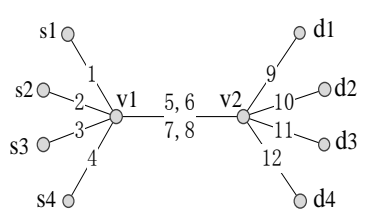

(b) weighted link schedule, $m=1$

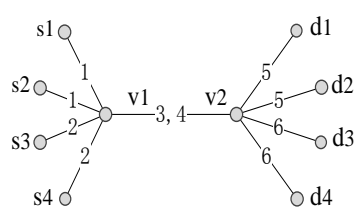

(d) weighted link schedule, $m=2$
Fig. 5. An illustrative example: unweighted link schedule is inefficient.

$\frac{2 a}{k(k+1)}$ bps. We should generalize the link scheduling that takes the traffic on each link into account.

Given a set of flows, denoted as $Q$, we adopt a certain routing algorithm to determine a routing path for each flow $f \in Q$. Let $|f|$ denote the rate at which data is sent from the source to the destination along that flow. The traffic load of any link $l$ is denoted as $\alpha(l)$, and is the sum of traffic generated by those flows passing through link $l$. That is, $\alpha(l)=\sum_{f \in Q: f \text { using } l}|f|$. We then assign a weight $w(l)=\alpha(l) / c(l)$ to each link $l$, which specifies the minimum number of required streams over link $l$ during the scheduling period $T$. Here, the maximum stream capacity $c(l)$ describes the largest achievable rate of one stream transmission over link $l$, which can be achieved if that stream uses the optimal sub-channel scheduled by SC model.

We show how a weighted link schedule can improve the network throughput using the example in Fig.5(d). By assigning the weight 1 to links $s_{i} v_{1}$ and $v_{2} d_{i}$ for $1 \leq i \leq k$ while $k / 2$ to link $v_{1} v_{2}$, obviously, $3 k / 2$ time slots can guarantee a feasible schedule. The total network throughput is now $2 a / 3$ bps and each flow has a throughput of $2 a / 3 k$ bps, being increased compared with the unweighted link schedule in an order of $k$. We also show how MIMO links can improve the network throughput by comparing examples shown in Fig.5(d) and Fig.5(b). Fig.5(b) illustrates a weighted link schedule where each node has only one antenna. The total network throughput is $a / 3 \mathrm{bps}$ and each flow has a throughput of $a / 3 k$ bps.

The challenge is how to derive a valid weighted link scheduling. In our previous scheduling algorithms, each active link $l$ is first assigned a time slot to transmit one data stream since its weight is one. For the traffic aware link scheduling, those active links are different in terms of their weights. Consequently, the basic procedures of our previous algorithms cannot address such a new issue well. Fortunately, our previous algorithms have been appended another step to allocate additional streams as many as possible for each scheduled link. We can take the traffic on each link into account at such a step. Specifically, the centralized algorithm processes scheduled links in the descending order of the weight of each link, while in the distributed algorithm, each link competes channel with a probability which is proportional to its weight. Such simple modifications will allocate links with high weight more data streams, so as to increase the capacity for links, which are shared by many flows. Links, whose weight is still 


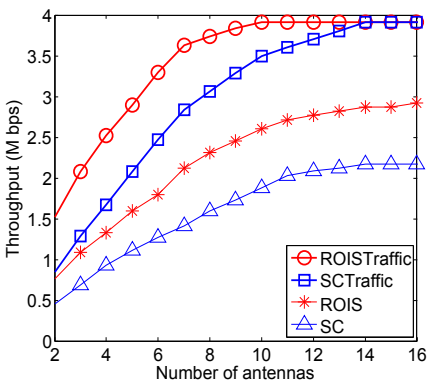

(a) Network throughput.

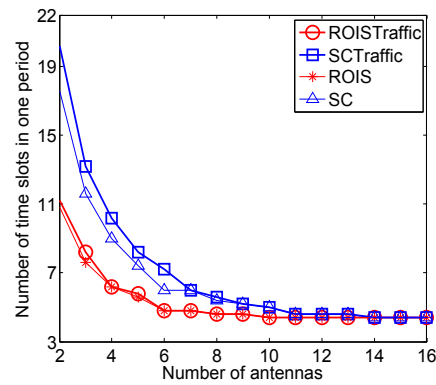

(b) Number of time slots in one period.

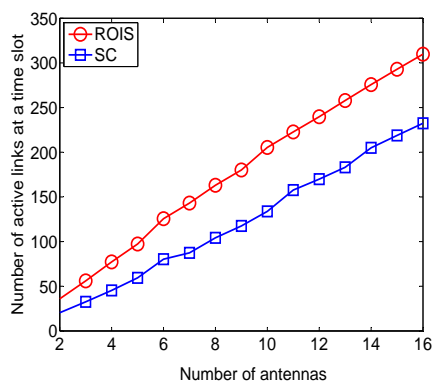

(c) Number of active links per time slot.

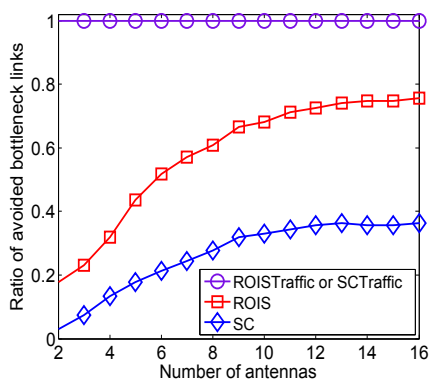

(d) Ratio of avoided bottleneck links.

Fig. 6. The impact of the number of antennas at each terminal under distributed algorithms.

larger than the number of allocated data streams, will be rescheduled by repeating the above process in the next time slot.

\section{Performance evaluation}

We start with the simulation methodology and scenarios and evaluate achievable throughput in multi-hop MIMO networks under different scheduling algorithms and network settings.

\subsection{Simulation methodology and scenarios}

Our simulations are based on SWANS++ that provides a good platform for simulation of wireless networks. We generate a random multi-hop MIMO network with 500 terminals. All terminals are uniformly distributed in a $800 \mathrm{~m} \times 800 \mathrm{~m}$ square. The evaluations are conducted under a Rayleigh fading MIMO channel, where the exponent for path loss formula is 2.8 and the shadowing deviation is 6.0. The default transmission power is $15 \mathrm{dBm}$. The default radio reception sensitivity is set to be $-72 \mathrm{dBm}$, so the initial interference range is $78 \mathrm{~m}$. The default radio reception threshold is $-67 \mathrm{dBm}$, and thus the transmission range is $51 \mathrm{~m}$. The maximum stream capacity $c(l)$ of each link $l$ to transmit one stream is set to unity (1024 bytes) so as to normalize the stream capacity, as used in the literature [14]. We randomly generate 100 pairs of source and destination nodes, and discover an end-to-end flow for each reachable pair of terminals using the DSR routing protocol [18].

Given a set of flows, denoted as $Q$, we assume the network delivers such flows in $T_{S}$ time slots under a schedule $S$, then the network throughput under $S$ is characterized by $\frac{\sum_{f \in Q}|f|}{T_{S}}$. For any given link $l$ passed by at least one of those flows, our traffic-aware scheduling has allocated $w(l)=\frac{\sum_{f \in Q: f \text { using }}|f|}{c(l)}$ streams to deliver its traffic load in $T_{S}$ time slots. These streams, however, usually differ in the stream gains no matter they are scheduled by the stream control $S C$ model or ROIS model, and cannot approach the maximum achievable rate $c(l)$. Actually, the capacity of each stream depends on the the power allocation and the sub-channel condition, and can be estimated at its transmitter during scheduling. Therefore, the real traffic load of link $l$ under $S$ should not exceed its link capacity, which is the sum of the stream capacities of its all streams. Assume link $l$ allocates its link capacity for all flows passing it equally, the rate at which data is sent over link $l$ for flow $f$ can be represented as the ratio of the link capacity to the number of flows passing link $l$. Consequently, the real value of $|f|$ is the minimum one among its allocated transmission rates of those links passed by flow $f$.

We first regulate three parameters to evaluate their impact on the performance of the proposed distributed link scheduling algorithms. They are the number of antennas, the average hop length of flows, and the interference range. The default number of antennas is 3 . The default ratio of the interference range to the transmission range is 1.5 . We then compare the network throughput under the distributed and centralized link scheduling algorithms with the SC and ROIS models. Let terms "ROISTraffic" and "SCTraffic" denote the traffic-aware link scheduling algorithms under the ROIS and SC models, respectively. We use other three metrics to explain the changes of the network throughput. They are the period $T$ of a feasible link schedule, the ratio of avoided bottleneck links, and the average number of links that can be active at one time slot.

\subsection{Impact of the number of antennas}

The number of antennas at each terminal varies from 2 to 16. We try to discover and schedule 100 end-to-end flows, but, DSR protocol only successfully responds only a few part of the flows. The average hop length of flows is 22.11. The largest link weight is 3 . The ratio of the interference range to the transmission range is the default value. Fig.6(a) shows that regardless the scheduling algorithms, the throughput first increases as the number of antennas increases, then reaches and keeps at the highest value after the number of antennas exceeds a threshold. The period of a schedule also stops decreasing simultaneously, as shown in Fig.6(b). The reason is that after the number of antennas reaches a threshold, for example 10 under such a setting, all constraints about the secondary interference have been satisfied. Thus, the radio constraint becomes the dominating factor, which is fixed as the number of antennas increases. In such a case, for any active link $l$ at a given time slot, it cannot be scheduled to an earlier time slot even the number of antennas increases continuously.

Fig.6(a) indicates that each algorithm based on the ROIS model outperforms the corresponding algorithm based on the SC model. The reason is that irrespective of the number of antennas, the ROIS model can allocate more links to be active at the same time slot than the SC model, as shown in Fig.6(c). The algorithms based on the ROIS model hence use less time slots to achieve a feasible schedule than that based on the SC model, as shown in Fig.6(b), and obtain a higher throughput. 


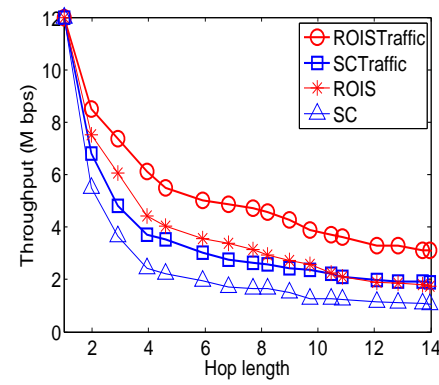

(a) Network throughput.

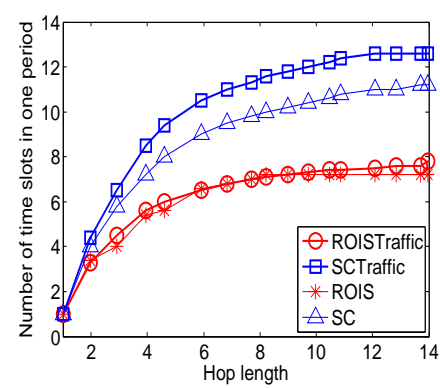

(b) Number of time slots in one period

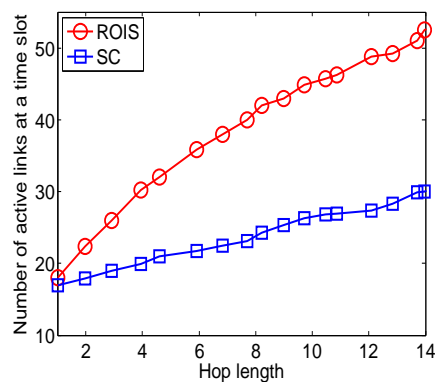

(c) Number of active links per time slot

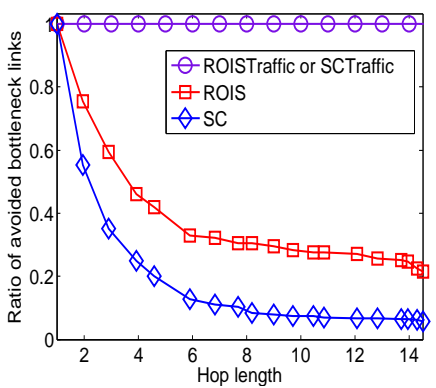

(d) Ratio of avoided bottleneck links.

Fig. 7. The impact of the average hop length under distributed algorithms.

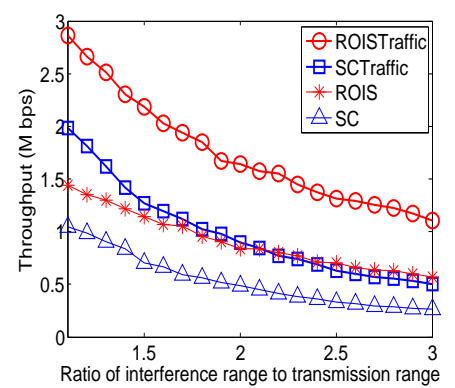

(a) Network throughput.

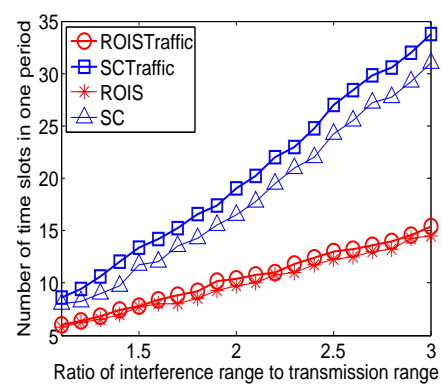

(b) Number of time slots in one period.

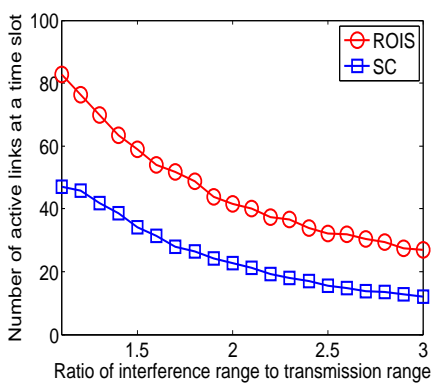

(c) Number of active links per time slot.

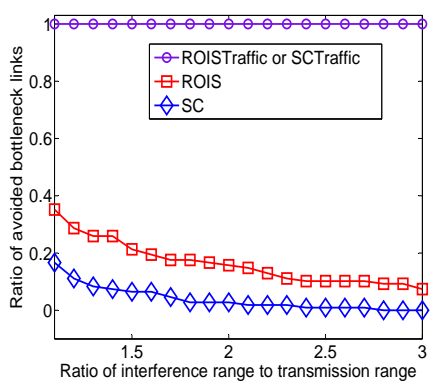

(d) Ratio of avoided bottleneck links.

Fig. 8. The impact of the interference range under distributed algorithms.

On the other hand, each traffic-aware algorithm outperforms the correlated standard algorithm although the scheduling period of the former is a little bit larger than the latter, as shown in Fig.6(b). The reason is that traffic-aware algorithms allocate sufficient time slots for those bottleneck links, which do not be addressed by the standard algorithms, as shown in Fig.6(d). Thus, the traffic-aware algorithms make some flows obtain higher transmission rates than the standard algorithms.

\subsection{Impact of the hop length}

In such a scenario, each flow is partitioned into a series of disjoint flows to be scheduled in each round, where the average hop length ranges from 1 to 15 . We consider 15 different hop lengths and generate random sets of flows for each hop length. The largest link weight among links is still 3. The number of antennas and the ratio of the interference range to the transmission range are the default values, respectively. Fig.7(a) shows that irrespective of the scheduling algorithms, the network throughput decreases as the average hop length increases. This can be explained as follows. First, the average out-degree and in-degree of nodes in an interference graph increase as the average hop length increases. Potentially there are more interfering links along the flows that need to be scheduled. Second, the longer the multi-hop flows, the more bottleneck links likely arise, as shown in Fig.7(d). Thus, more time slots are required for a feasible schedule, as shown in Fig.7(b), hence resulting in a reduced throughput.

Fig.7(c) shows that the average number of active links per time slot increases as the average hop length increases although the number of antennas is fixed. Fig.7(d) shows that the ratio of avoided bottleneck flows decreases as the hop length increases. More bottleneck flows arise while each active link has less chance to append additional streams due to more out-neighbors. These findings can explain why the throughput decreases as the hop length increases.

\subsection{Impact of the interference range}

The average hop length of flows is 22.11. The largest link weight among links is 3 . The number of antennas is the default value. In such simulations, we vary the ratio of the interference range to the transmission range from 1.1 to 3. Fig.8(a) shows that irrespective of the scheduling algorithms, the network throughput decreases as the interference range increases. This result can be explained as follows. The increase of interference range directly increases the probability of interference in the vicinity. As shown in Fig.8(c), less links can then satisfy the scheduling conditions defined in Section 4. As a result, more time slots are required for a feasible schedule, as shown in Fig.8(b). Similarly, each link has less chance to append other streams at the same time slot after it is assigned a given time slot. Thus, less bottleneck links are addressed as the interference range increases, as shown in Fig.8(d).

\section{ReLATED WORK}

There are three spatial reuse schemes, namely spatial reuse with stream control, spatial reuse with non-stream control, and spatial reuse with interference avoidance. As we have discussed in Sections 1 and 2.3, stream control-based schemes generally outperforms the other two schemes. In this section, we compare related works with our work, with respect to how the spatial reuse schemes are applied and utilized.

$\mathrm{Chu}$ et al. propose a cross-layer scheduling algorithm to fully exploit the multiuser diversity and spatial diversity [9]. 
That algorithm achieves similar benefits as the stream control scheme does. A better network throughput can be achieved if such an algorithm is incorporated with an interference avoidance scheme. Sundaresan et al. propose a heuristic scheduling algorithm called SCMA to exploit the gain of stream control [17]. Wang et al. derive an upper bound of the network capacity using stream control-based scheduling [6]. They further propose a scheduling algorithm that makes the resultant network capacity be close to the derived upper bound. Mundarath et al. propose a specific MAC protocol to realize both spatial multiplexing and spatial reuse [15] such that each terminal seamlessly allocates its available DOFs to either spatial multiplexing or spatial reuse. A common point of such related works is that they utilize stream control to schedule links. Our study reveals that the gain of spatial reuse can be further improved if interference avoidance is also applied to increase the concurrent number of transmissions.

Hamdaoui et al. study three protocols for multi-hop MIMO networks, SMP, SRMP, and SRP [14]. SMP and SRMP are TDMA-based spatial multiplexing and spatial reuse, respectively. SRP is a special case of spatial reuse in which each active link delivers only one stream. The preferred interference model, called CiM, requires either the transmitter or the receiver be responsible for spatial reuse. If the transmitters suppress interference, CiM is just a case of spatial reuse with interference avoidance, hence offering less network throughput than the ROIS model. If the transmitters adopt stream control and the receivers are responsible for suppressing interference, $\mathrm{CiM}$ achieves at most the same throughput as stream control.

TDMA-based Link scheduling is another class of approaches to ensure networking throughput. It's usually modeled as a vertex coloring problem in conflict graphs, which cannot utilize the advantages of MIMO links. In this paper, we propose two dedicated link scheduling algorithms. Link scheduling based on the SC model achieves equivalent network throughput as the existing link scheduling ones like [6], [17] with the stream control model. Our analysis and simulation results demonstrate that the link scheduling based on the ROIS model outperforms those related works based on the SC model.

\section{Conclusion}

This paper presents our study on efficient link scheduling for multi-hop MIMO networks. We use two dedicated interference suppression models to fully utilize the unique characteristics of MIMO links. Based on these models, both centralized and distributed link scheduling algorithms are devised to maximize the network throughput. For better applicability in practice, we further improve the proposed algorithms to address the nonuniform traffic demands. The comprehensive evaluations show that the ROIS-based link scheduling outperforms the SC-based link scheduling in terms of the network throughput.

\section{ACKNOWLEDGMENTS}

The authors would like to thank anonymous reviewers for their constructive comments. The work is partially supported by the NSFC under Grants Nos. 61170284, 60903206, 61003277, and 61170213. The research of Yunhao Liu is supported in part by the NSFC Major Program under Grant No. 61190110 and the National High-Tech R\&D Program of China (863) under Grant No. 2011AA010100. The research of Xiang-Yang Li is partially supported by the NSF CNS-0832120, the NSF CNS-1035894, the NSFC under Grant No. 61170216, the China 973 Program under Grant No. 2011CB302705, and the Zhejiang Provincial Key Innovative Research Team program for Zhejiang Provincial Overseas High-Level Talents.

\section{REFERENCES}

[1] M. Vu and A. Paulraj, "MIMO wireless linear precoding," IEEE Signal Processing Magazine, vol. 24, no. 5, pp. 86-105, 2007.

[2] E. Gelal, K. Pelechrinis, T.-S. Kim, I. Broustis, S. V. Krishnamurthy, and B. Rao, "Topology control for effective interference cancellation in multi-user mimo networks," in Proc. IEEE INFOCOM, San Diego, CA, USA, Mar. 2010.

[3] H. Dai, A. F. Molisch, and H. V. Poor, "Downlink capacity of interference-limited MIMO systems with joint detection," IEEE Transactions on Wireless Communications, vol. 3, no. 2, pp. 442-453, 2004.

[4] W. Rhee and J. M. Cioffi, "On the capacity of multiuser wireless channels with multiple antennas," IEEE Transactions on Information Theory, vol. 49, no. 10, pp. 2580-2595, 2003.

[5] S. Chu and X. Wang, "Mimo-aware routing in wireless mesh networks," in Proc. 29th IEEE INFOCOM, San Diego, CA, USA, 2010, pp. 2784 2792.

[6] Y. Wang, D. M. Chiu, and J. C. Lui, "Characterizing the capacity gain of stream control scheduling in MIMO wireless mesh networks," Wireless Communications and Mobile Computing, vol. 9, no. 6, pp. 819-829, 2009.

[7] S. Chu and X. Wang, "Adaptive exploitation of cooperative relay for high performance communications in mimo ad hoc networks," in Proc. 7th IEEE MASS, San Francisco, CA, USA, 2010, pp. 21-30.

[8] K. Sundaresan, R. Sivakumar, M. A. Ingram, and T. Y. Chang, "A fair medium access control protocol for ad-hoc networks with MIMO links," in Proc. 23th IEEE INFOCOM, Hong Kong, Mar. 2004.

[9] S. Chu and X. Wang, "Opportunistic and cooperative spatial multiplexing in mimo ad hoc networks," IEEE/ACM Transactions on Networking, vol. 18, no. 5, pp. 1610-1623, 2010.

[10] J. S. Jiang, M. F. Demirkol, and M. Ingram, "Measured capacities at $5.8 \mathrm{GHz}$ of indoor MIMO systems with MIMO interference," in Proc. IEEE VTC, 2003, pp. 388-393.

[11] M. F. Demirkol and M. A. Ingram, "Control using capacity constraints for interfering MIMO links," in Proc. 13th IEEE PIMRC, 2002, pp. 1032-1036.

[12] S. Gaur, J. S. Jiang, M. A. Ingram, and M. F. Demirkol, "Interfering MIMO links with stream control and optimal antenna selection," in Proc. IEEE GLBOCOM, Dallas, TX, Nov. 2004, pp. 3138-3142.

[13] S. Gaur and M. Ingram, "Stream control for interfering MIMO links with linear MMSE receivers," in Proc. 41th Annual Asilomar Conference on Signals, Systems, and Computers, California, USA, Nov. 2007, pp. 1664-1668.

[14] B. Hamdaoui and K. G. Shin, "Characterization and analysis of multihop wireless MIMO network throughput," in Proc. the 8th ACM Mobihoc, Quebec, Canada, 2007, pp. 120-129.

[15] J. C. Mundarath, P. Ramanathan, and B. D. V. Veen, "Exploiting spatial multiplexing and reuse in multi-antenna wireless ad hoc networks," Elsevier Journal of Ad Hoc Networks, vol. 7, no. 2, pp. 281-293, 2009.

[16] K. Sundaresan, R. Sivakumar, M. A. Ingram, and T. Y. Chang, "Medium access control in ad hoc networks with mimo links: Optimization considerations and algorithms," IEEE Transactions on Mobile Computing, vol. 3, no. 4, pp. 350-365, 2004.

[17] K. Sundaresan and R. Sivakumar, "A unified mac layer framework for ad-hoc networks with smart antennas," IEEE/ACM Transactions on Networking, vol. 15, no. 3, pp. 546-559, 2007.

[18] X. Chen, J. Shen, and J. Wu, "A novel information model for efficient routing protocols in delay tolerant networks," in Proc. 23th IPDPS, Rome, Italy, 2009, pp. 1-8.

[19] W. Wang, X. Li, O. Frieder, Y. Wang, and W. Z. Song, "Efficient interference-aware TDMA link scheduling for static wireless networks," in Proc. 12th ACM MOBICOM, Los Angeles, USA, 2006, pp. 262-273.

[20] L. Gao, X. Wang, and Y. Xu, "Multi-radio channel allocation in multihop wireless networks," IEEE Transactions on Mobile Computing, vol. 8, no. 11 , pp. 1454-1468, 2009. 


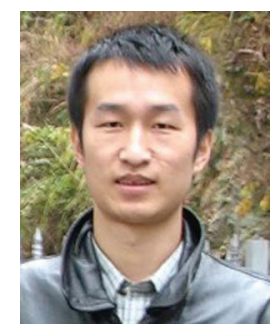

Deke Guo received the B.S. degree in industrial engineering from Beijing University of Aeronautic and Astronautic, Beijing, China, in 2001, and the Ph.D. degree in management science and engineering from National University of Defense Technology, Changsha, China, in 2008. He is now an associate professor of Information System and Management, National University of Defense Technology, Changsha, China. His current research interests include peer-to-peer computing, wireless networks, data center networking, Bloom filters, and interconnection networks. He is a member of the ACM and the IEEE.

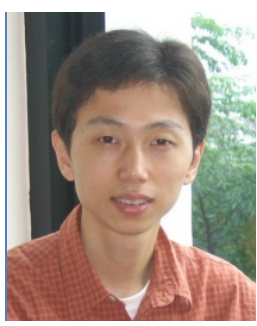

Yuan He Yuan He received his BE degree in Department of Computer Science and Technology from University of Science and Technology of China in 2003, and his PhD degree in Department of Computer Science and Engineering, Hong Kong University of Science and Technology, in 2010 . He is now a postdoc fellow at TNLIST and School of Software, Tsinghua University. His research interests include peer-topeer computing, sensor networks, and pervasive computing. He is a student member of the IEEE and the IEEE Computer Society.

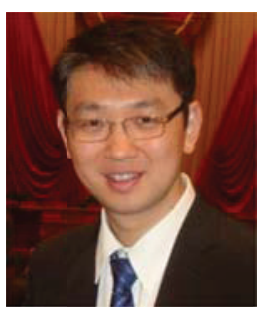

Yunhao Liu received the BS degree in automation from Tsinghua University, China, in 1995 , the MS and PhD degrees in computer science and engineering from Michigan State University, in 2003 and 2004, respectively. He is now EMC Chair Professor at Tsinghua University, as well as a faculty member with the Hong Kong University of Science and Technology. His research interests include wireless sensor network, peerto-peer computing, and pervasive computing. $\mathrm{He}$ is a senior member of the IEEE Computer Society and an ACM Distinguished Speaker.

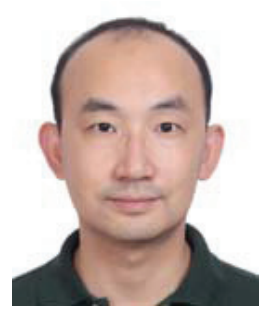

Xin Wang received the Ph.D. degree in information and communication engineering from the National University of Defense Technology, Changsha, China, in 2008. He is now an assistant professor of Electronic Science and Engineering, National University of Defense Technology, Changsha, China. His current research interests include signal processing for broadband MIMO and cooperative communications. $\mathrm{He}$ is a member of the IEEE.

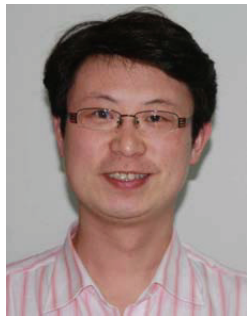

Panlong Yang received his BS degree, MS degree, and $\mathrm{PhD}$ degree in communication and information system from Nanjing Institute of Communication Engineering, China, in 1999, 2002, and 2005 respectively. During November 2006 to March 2009, he was a postdoc fellow in the Department of Computer Science, Nanjing University. Dr. Yang is now an associate professor in the Nanjing Institute of Communication Engineering, PLA University of Science and Technology. His research interests include wireless mesh networks, wireless sensor networks and cognitive radio networks. $\mathrm{He}$ has also served as a member of program committees for several international conferences. He is a member of the IEEE Computer Society and ACM SIGMOBILE Society.

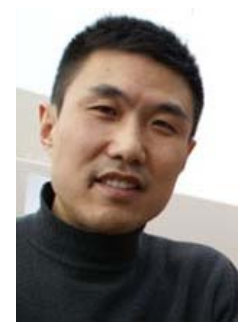

Xiang-yang $\mathbf{~} \mathbf{i}$ is a professor of Computer Science at the Illinois Institute of Technology. He received MS (2000) and $\mathrm{PhD}$ (2001) degree at Computer Science from University of Illinois at Urbana-Champaign, B.Eng. at Computer Science and Bachelor degree at Business Management from Tsinghua University, P.R. China in 1995. His research interests include cyber physical systems, wireless networks, social networks, and security. He is an editor of "IEEE TPDS", "IEEE TMC", "Networks: An International Journal", "Ad Hoc \& Sensor Wireless Networks: An International Journal". In 2008, he published a monograph "Wireless Ad Hoc and Sensor Networks: Theory and Applications", by Cambridge University Press. He is a senior member of the IEEE and a member of ACM. 